\title{
Red Eye: Next Steps for Conducting Research in Knowledge, Attitude and Practice in Ophthalmology
}

Francisco J. Bonilla-Escobar', Hugo H. Ocampo-Dominguez ${ }^{1,2}$.

\begin{abstract}
.
Background: Research in Knowledge, Attitude and Practice (KAP) in health sciences is relevant to health care providers and patients to identify factors to address educational interventions. Methods: A pilot study based on surveys amongst participants in a medical update conference in Cali, Colombia, was conducted to estimate participants' knowledge on red eye in 2011. Results: The population was composed of medical students and general practitioners, with $72.7 \%$ of students being in their final year of their training. The classification of red eye was correct in $47 \%$ of respondents and we found errors in the classification of emergency, glaucoma and uveitis. Conclusions: Further research proposals in KAP are required for the recognition of this medical education indicator.
\end{abstract}

Keywords: Health Knowledge, Attitudes, Practice; Eye Hemorrhage; Students, Medical (Source: MeSH, NLM).

About the Author

Francisco J. Bonilla-Escobar is a recent graduate of the Universidad del Valle, Cali, Colombia and currently a Master's student in Epidemiology at Universidad del valle as well as Research As sistant at CISALVA Institute. He is former President of the Colombian Society of Medical Students' Associations (ASCEMCOL).

Submission: 2012, April 30. Accepted: 2012, Jul 11.

Process: Peer-Reviewed.

\section{Introduction.}

A study of knowledge, attitudes and practices (KAP) has been widely used in community medicine and in some clinical areas, an annual increase of publications in the area in the past 10 years, illustrating its importance.'

The relevance of KAP studies lies in the possibility of the researcher to recognize, measure and establish the extent of knowledge on a specific topic in a population. It's commonly used as a tool to provide an educational diagnosis of health staff on diseases and their medical or surgical management, enabling to design interventions for improving the care of patients, and in medical education. ${ }^{2}$

It is striking the lack of these studies in ophthalmology as evidenced in MEDLINE by searching PubMed (http:// www.ncbi.nlm.nih.gov/sites/entrez) using the term "Health Knowledge, Attitudes, Practice"[Mesh] appear as result 58,163 articles, but adding the term "Ophthalmology" ("ophthalmology"[Mesh] AND "Health Knowledge, Attitudes, Practice"[Mesh]) appear a total of $55(0.094 \%)$, of which 13 do not correspond to KAP studies in ophthalmology, leaving $42(0.072 \%)$ articles in total, of which $15(35.7 \%)$ assessed patients, $20(47.6 \%)$ ophthalmologists, $3(7.1 \%)$ general practitioners, $3(7.1 \%)$ medical students and $1(2.3 \%)$ tested two populations: ophthalmologists and patients.

From this information grew the initiative to propose a pilot study based on a self-administered questionnaire assessing basic knowledge in a frequent ocular sign in general practice, the red eye; listed as the leading cause of eye consultation to the general practitioner in primary care. ${ }^{3}$

Red eye is the most common and nonspecific ocular sign encountered in emergency and outpatient settings. The term red eye might represent infectious and inflammatory conditions involving one or more ocular tissues, including the conjunctiva, cornea, lids, and internal ocular structures. $^{3}$

This symptom accounts for approximately $15 \%$ of consultations for ophthalmologists and $6 \%$ for general medical practitioners. ${ }^{4}$ Red eye is the most common ocular symptom seen by primary care physicians ${ }^{5}$ and therefore the knowledge, attitudes and practices is important to guide the patient management and improve the visual prognosis of patients. The causes of red eye are show in Table 1.

The objective of this study was to establish the Knowledge in red eye, of participants in a medical update conference in Cali, Colombia.

\section{Methods}

A pilot study based on surveys with a self-administered questionnaire amongst participants in a medical update conference in Cali, Colombia, was conducted in order to estimate knowledge on the approach to red eye and its 
differential diagnosis. The survey consisted of five multiple choice questions with a single correct answer, evaluating the following topics: classification of red eye, clinical presentation of acute angle closure glaucoma, conjunctivitis and uveitis, and the recognition of an ophthalmologic emergency. The survey counted with the review and approval of 2 experts in the field.

The study population was the participants attending a medical update conference held in the Cali, Colombia in September 2011, which involved 202 individuals, who were medical students and general practitioners.

The study is considered of "minimal risk" as there is no intervention and the individual's identity is kept anonymous, fulfilling the ethical standards issued by the Nuremberg Code and the Declaration of Helsinki on research in humans.

The surveys were collected and typed in a database designed for this purpose in Microsoft office Access ${ }^{\circledR}$, and we searched for typing errors by evaluating $20 \%$ of the surveys. Data analysis consisted on a frequency analysis with statistical software Stata $12^{\circledR}$, presenting quantitative variables with means and standard deviation (SD), and categorical variables in frequencies and percentages.

\section{Results.}

Two hundred and two surveys were distributed to the participants. The response rate was $32.7 \%$ (66).

Among people who completed the survey, 26 (39.39\%) were female and $40(60.61 \%)$ were men. The ages of participants was in the range of 18 to 37 years with a mean of 23.6 (SD \pm 3.7). Eighty percent (53) of responders were in the range of 18 to 25 years and $19.7 \%$ (13) were between 26 to 37 years.

The participants were from universities or medical institutions, with a total of $11(16.67 \%)$ general practitioners and $55(83.3 \%)$ medical students from 9 different universities.
Table 1. Common causes of red eye. Adapted from Seth (3).

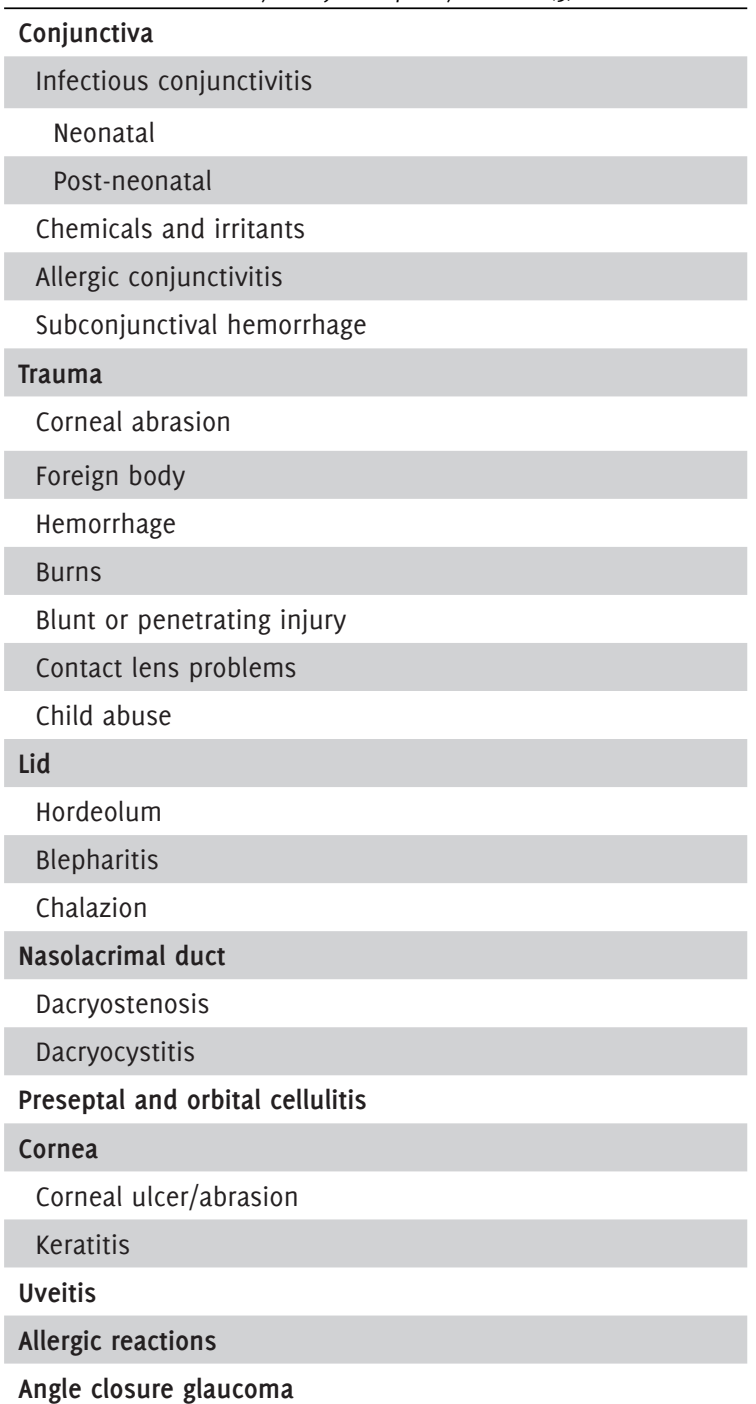

We found that, $25(37.88 \%)$ persons were in their 5 th year of medical school of a 6-year program, $13(19.7 \%)$ final year medical students, 11 (16.67\%) general practitioners, $10(15.15 \%)$ in 4 th year of medical school, $6(9.09 \%)$ in 3 rd

Figure 1. Distribution of survey responses about knowledge in red eye classification, photophobia sign/symptom, and eye emergency, of participants in a medical update congress in Cali, Colombia.

Red Eye Classification

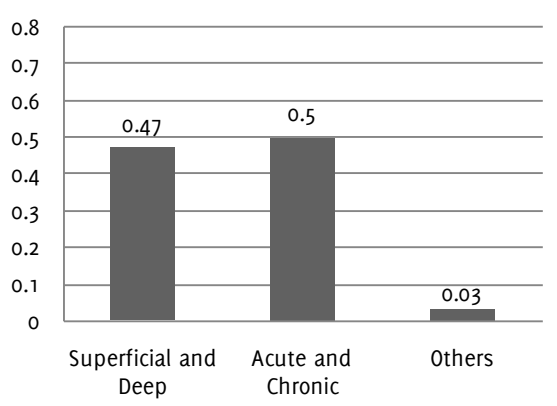

Photophobia is a sign of...

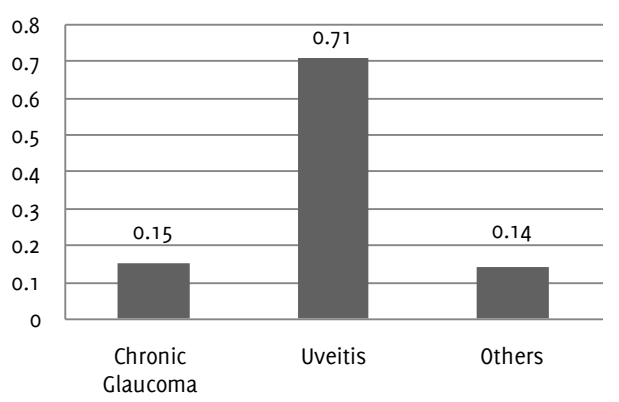

Eye Emergency

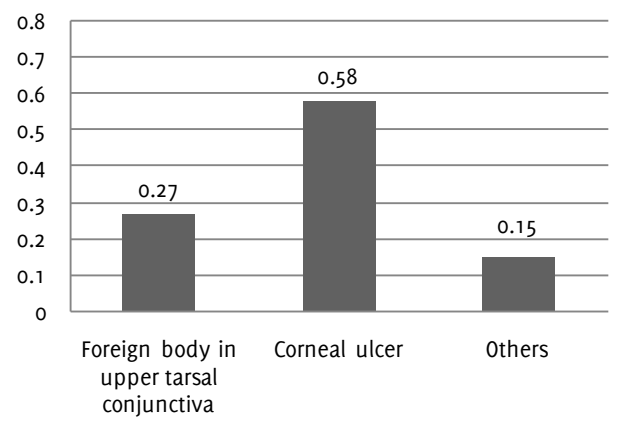


year, and $1(1.52 \%)$ person in 1st year of medical school. Majority of the universities in the country were represented by medical students in their final years (4th to 6 th), with percentages of students at this academic level in each university ranging from 50 to $100 \%$.

The correct answers were distributed as follows: in the classification of red eye $47 \%$ (31), in the clinical presentation of acute angle closure glaucoma $95.5 \%(63)$, in the case of conjunctivitis $93.9 \%(62)$, in uveitis $71.2 \%(47)$, and in recognition of an ophthalmological emergency $57.6 \%$ (38) of participants gave a correct answer.

Additionally we found wrong concepts in some answers (Figure 1): In the classification of red eye, the 50\% (33) of the participants chose the acute and chronic classification. About the clinical presentation of uveitis, $28.7 \%$ (19) of the participants answered incorrectly and the most frequent mistake was diagnosing chronic glaucoma (open angle glaucoma) with a $15.1 \%$ (10). Finally, about an ocular emergency, $57.6 \%$ (44) of participants answered incorrectly, and $27.3 \%$ (18) considered a retained foreign body in upper tarsal conjunctiva as an emergency.

\section{Discussion.}

The Knowledge possessed by a community refers to their understanding of that topic. Attitude refers to their feelings toward this subject, as well as any preconceived ideas they may have towards it. Practice refers to the ways in which they demonstrate their knowledge and attitudes through their actions. ${ }^{2}$

To consider the red eye as a sign should be the first teaching for medical students in their approximation to ophthalmology, but this is usually neglected because the practice in this area is inadequate; in Colombia for example, the regular rotation in most universities lasts only two weeks.

Probably the red eye is not the most common consultation in general practice but when the physician is exposed to it, he should be able to provide a differential diagnosis and propose a management plan or at least determine emergency situation from non-emergency referral. ${ }^{4}$

The pathophysiology of the red eye relies in the ocular anatomy and physiology. The vasculature of the eye is comprised of a superficial and deep layers, the first nurturing the conjunctiva and episclera, and the second the choroid and retina, therefore a problem in these vasculatures anywhere in the eye will produce a red eye,${ }^{6}$ for this reason it is not a diagnosis, it is a sign, and the challenge lies in identifying the precise diagnosis.

The history plays a fundamental role in the diagnosis of a disease manifested with red eye, and the visual acuity needs to be evaluated as it is the key to provide an accurate initial assessment and differential diagnosis. ${ }^{4,6}$

The co-findings of red eye are multiple and include redness, epiphora, pain, photophobia, itching, and visual acuity alteration. Recognition of situations when a red eye constitutes an emergency is fundamental in the primary care setting for the initial treatment and referral of the indicated patients, preventing the possibilities of permanent loss of vision. ${ }^{-4,46}$

The main indication for referral of patients to an ophthalmologist are: vision loss, purulent discharge, corneal ede$\mathrm{ma}$, opacities and staining with fluorecein, history of trauma with anatomical distortion, or distorted pupil. ${ }^{3}$ Other authors also suggest that if the symptoms are severe or rapidly progressive, urgent referral to an ophthalmologist is appropriate. ${ }^{6}$

Petricek et al., ${ }^{4}$ conducted a survey to estimate the percentage of patients presenting with red eye and to examine differential diagnosis and treatment amongst general practitioners and ophthalmologists from 9 countries in Eastern Europe and the Middle East. They found that patients presented with chief complaint of red eye in $15 \%$ of the ophthalmologist practice and $6 \%$ of general practitioners. Diagnosis of allergic conjunctivitis was the most common cause of red eye $(35 \%)$, followed by dry eyes $(25 \%)$ and bacterial conjunctivitis $(24 \%)$, and the general practitioners were more likely to prescribe combinations of topical antibiotic and steroids.

In this pilot study it is concerning that a significant percentage of medical students in their final years of medical education are unclear of the real causes of ocular urgencies. It's important to evaluate the medical education in ophthalmology because the general practitioners are who have the first possibility to direct the management of an urgent case, which will impact the visual prognosis. ${ }^{4}$

Further studies on KAP in ophthalmology are required, since they account for $0.07 \%$ of the articles published in this topic in PubMed, and this report found errors in the evaluated population in the classification of diseases where the main symptom is red eye. The authors propose further investigation in KAP in red eye and other eye diseases is needed, to improve medical education and the patient management.

This study has limitations in sample size and sample selection, and in administering the same questionnaire to people in different stages of their training, but the assessment results and the small number of publications in KAP in ophthalmology, are an incentive and serves to encourage the implementation of further research about KAP in ophthalmology and to highlight the importance of such investigations. 


\section{Short Communications}

\section{References.}

1. Viedma Gil De Vergara P, Arranz Lázaro M, García León C, Soto Torres B. Utilización de cuestionarios en los estudios que miden conocimientos/ actitudes/prácticas: referencias bibliográficas incluidas en Medline (1994-1998). Gac Sanit 1999;13(91):8143.

2. Kaliyaperumal K. Guideline for Conducting a Knowledge, Attitude and Practice (KAP) Study. AECS Illumination 2004;4(1):7-9.

3. Seth D, Khan Fl. Causes and Management of Red Eye in Pediatric Ophthalmology. Curr Allergy Asthma Rep 2011;11:212-219.

4. Petricek I, Prost M, Popova A. The differential diagnosis of red eye: a survey of medical practitioners from Eastern Europe and the Middle East. Ophthalmologica 2006;220:229-37.

5. Granet D. Allergic rhinoconjunctivitis and differential diagnosis of the red eye. Allergy Asthma Proc 2008;29:565-574.

6. Khare GD, Andrew-Symons RC, Do DV. Common Ophthalmic emergencies. Int J Clin Pract 2008;62(11):1776-84

Conflict of Interest Statement at Funding

The Authors have no funding, financial relationships or conflicts of interest to disclose.

Cite as:

Bonilla-Escobar FJ, Ocampo-Dominguez HH. Red Eye: Next Steps for Conducting Research in Knowledge, Attitude and Practice in Ophthalmology. Int J Med Students 2013;1(1):24-7. 